\title{
Erfahrungen mit den A-IQI Qualitätsindikatoren in Österreich
}

\author{
F. Fuchs \\ Qualitätsmanagement \\ Schlüsselvwörter \\ Qualitätsmessung \\ Kodierqualität \\ Deer-Review-Verfahren \\ Keywords \\ quality measurement \\ coding quality \\ peer review
}

Institut

Niederösterreichische Landeskliniken-Holding, St. Pölten, Österreich

Bibliografie

Dol $10.1055 / \mathrm{s}-0031-1286077$ Dtsch Med Wochenschr 2011; 136: S48 - (c) Georg Thieme Verlag KG Stuttgart · New York . ISSN 0012-0472

Korrespondenz Dr. Fabiola Fuchs

Leiterin der Abteilung Strat. Qualitätsentwicklung Niederösterreichische Landeskliniken-Holding Stattersdorfer Hauptstraße 6/C A-3100 St. Pölten Tel. +43 (0)2742313813 eMail fabiola.fuchs@ holding.Iknoe.at

\section{Ausgangslage}

$\nabla$

2009 wurden die bestehenden „German Inpatient Quality Indicators“ (Version 2.5) zwischen dem deutschen „Diagnosis Related Groups“ (DRG)-System und der österreichischen Leistungsorientierten Krankenanstaltenfinanzierung abgeglichen, woraus die „Austrian Inpatient Quality Indicators“ (A-IQI) entstanden sind. Seither ist das Indikatorenset innerhalb der Niederösterreichischen Landeskliniken-Holding (NÖLKH) in Verwendung. Ende 2009 fand ebenfalls das erste Peer-Review-Verfahren statt. Durch die Zusammenarbeit mit der Initiative Qualitätsmedizin ( $\mathrm{IQ}^{\mathrm{M}}$ ) konnten im März 2011 die ersten Reviewer-Schulungen mit 20 Primarärzten stattfinden und das gesamte Verfahren an deren Standard angepasst werden. Für das gesamte Jahr 2011 sind 22 Reviews geplant.

Die praktische Anwendung der Kennzahlen erfolgt in drei Schritten:

1. Messung

2. Interne Transparenz

3. Ergebnisbearbeitung.

Die Ergebnisse werden quartalsweise allen Managementebenen zur Verfügung gestellt. Die Ergebnisbearbeitung erfolgt in den Medizinischen Gremien (Fachbeiräte, Regionale Medizinische Beiräte) und in den Peer-Review-Verfahren [1].

\section{Erste Ergebnisse der Peer-Review-Verfahren} $\nabla$

Für die Auswahl der Peer-Review-Themen 2011 galten folgende Kriterien: die Berücksichtigung nur jener Fächer mit ausgebildeten Reviewern und das Ergebnis „NÖ Gesamt“ außerhalb des Zielbereiches. Für die Wahl der Kliniken wurden Konfidenzintervalle in Form eines Ampelsystems in das bestehende Auswertungstool eingearbeitet, um den PrimarärztInnen eine objektive Entscheidung präsentieren zu können. Besonders in den ersten Jahren ist eine transparente Darlegung der Auswahlkriterien für die Akzeptanz des Verfahrens von besonderer Wichtigkeit.

In den bisher durchgeführten Reviews zeigten sich, wie auch bereits bei den IQM-Peer-ReviewVerfahren, Verbesserungsmöglichkeiten in der ärztlichen Dokumentation, der Kodierqualität im Bereich der Hauptdiagnose und in der Einhaltung von aktuellen Leitlinien. Bei den konservativen Indikatoren ist die Kodierqualität insge- samt gesehen noch nicht zufriedenstellend. Um diese schnellstmöglich zu verbessern, wird in den nächsten zwei Monaten eine umfangreiche Kodierüberprüfung veranlasst. Des Weiteren erstellen Fachbeiratsmitglieder Kodierrichtlinien für bestimmte Erkrankungen (z.B. Herzinfarkt, Schlaganfall, transitorische ischämische Attacke).

\section{Nationale Anwendung $\nabla$}

Im April 2011 beschloss die Bundesgesundheitskommission die nationale Anwendung der A-IQI und des Peer-Review-Verfahrens. Der Einsatz der einheitlichen Ergebnisqualitätsmessung auf Basis von Routinedaten stellt einen Meilenstein im österreichischen Krankenhauswesen dar. Die Kennzahlenergebnisse sowie die Auswahl der Peer-Review-Themen werden innerhalb einer Steuerungsgruppe unter dem Vorsitz des Bundesministeriums für Gesundheit (BMG) und unter Beteiligung aller Fonds diskutiert. Im Zuge der Umsetzung wird den AnwenderInnen seitens des BMG ein einheitliches EDV-Auswertungstool zur Verfügung gestellt. Die ersten Schulungen und Review Verfahren sind für 2012 geplant.

\section{Weitere Vorgehensweise in der NÖLKH}

Die NÖLKH hat bereits im Sommer die Mitgliedschaft bei der $\mathrm{IQ}^{\mathrm{M}}$ beantragt, unter anderem deshalb, um sich aktiv in die Weiterentwicklung des Verfahrens einbringen und internationale PeerReview-Verfahren durchführen zu können. Ein weiterer Schritt für 2012 ist die Einbindung der QualitätsmanagerInnen in das Peer-Review-Verfahren, um die Abteilungsleitungen vor Ort bei der Umsetzung der Verbesserungsmöglichkeiten zu unterstützen. Außerdem ist die Weiterentwicklung der A-IQI auf die neueste Version G-IQI 3.1 geplant, damit auch künftig die internationale Vergleichbarkeit gewährleistet bleibt.

Autorenerklärung: Die Autorin erklärt, dass keine relevanten finanziellen Verbindungen in Bezug auf dieses Manuskript bestehen.

\section{Literatur}

1 Amon M, Fuchs F. Das Peer Review Verfahren. Die standardisierte Vorgehensweise der Niederösterreichischen Landeskliniken-Holding am Vorbild der Initiative Qualitätsmedizin zur Bearbeitung der Austrian Inpatient Quality Indicators Version 1.0. Edition Donau-Universität. Krems: 2011: 23 